\title{
Associations of alleles of microsatellite markers with agronomical traits of modern bread winter wheat varieties in Southern Ukraine
}

\author{
0. 0. Kolesnyk ${ }^{*}$, 0. M. Khokhlov, S. V. Chebotar
}

The Plant Breeding and Genetics Institute - National Center of Seed and Cultivar Investigations, 3, Ovidiopolska doroha st., Odesa, 65036, Ukraine, "e-mail: emerald-olga@ukr.net

Purpose. Defining marker-trait associations of microsatellite markers with specific regions of the genome that control important agronomical traits in the investigated varieties originated in the Plant Breeding and Genetics Institute - National Center of Seed and Cultivar Investigations and entered into the State register of plant varieties suitable for dissemination in Ukraine during different years. Methods. Molecular genetic methods (extraction of genomic DNA, polymerase chain reactions (PCR), electrophoresis of amplification products in polyacrylamide gel), field methods (phenological observations of heading date and analysis of plant height, visual assessment of the colour and length of wheat ear and awns), statistical methods (evaluation of trait means by descriptive statistic instruments of EXCEL package, ANOVA method performed by GLM instrument from AGROBASE 21 package). Results. During four growing years $(2010 / 11,2011 / 12,2012 / 13,2013 / 14), 47$ bread winter wheat varieties were phenotypically measured and analyzed with 17 microsatellite loci. 35 marker-trait associations (MTA) for heading date, 39 for plant height, 33 for awn size, 20 for ear colour and 8 for ear size were found to be stable and significant during two-four different growing years. Conclusions. Microsatellite markers that showed substantial and stable during different growing years associations with agronomical traits can be useful and suitable for marker-assisted selection (MAS) in Ukrainian wheat breeding programs.

Keywords: marker-trait associations, Triticum aestivum L., bread winter wheat varieties.

\section{Introduction}

Bread wheat (Triticum aestivum L.) is one of the most important and widely grown food crop in many countries, and therefore the main task of breeding programs all over the world is to increase grain yield (GYLD) of wheat. Basic components of wheat yield include such breeding traits as ear number per plant, grain number per ear and grain weight [1]. Grain size and (or) weight is the target for breeding not merely as a component of grain yield, but also because of its impact on flour yield and protein content, that also affect milling and baking quality [2]. Some other agronomic traits, including anthesis date, plant height $(\mathrm{PH})$ and grain volume weight, also have an effect on GYLD [3]. The efforts of breeders to increase yield are of great significance as they have to feed the expanding mass of human population taking into account the constantly changing market requirements. Such efforts focused on the selection of de-

Olga Kolesnyk

http://orcid.org/0000-0003-0603-5311

Alexander Khokhlov

http://orcid.org/0000-0003-4459-195X

Sabina Chebotar

http://orcid.org/0000-002-9130-7272 sired genotypes to develop high-yielding varieties are implemented in Ukraine where wheat breeding began more than 100 years ago. For more than one century, the South of Ukraine has been regarded as the main bread winter wheat producing region of the country due to its rich black soils and a relatively dry climate. The special approach to breeding that was applied at the Plant Breeding and Genetics Institute (PBGI) [4] included traditional and nontraditional methods such as anther culture, biochemical and molecular markers, resulted in the release of new winter wheat varieties with high yield potentials and well expressed adaptation traits. Nowadays, a great progress in a conventional breeding is enhanced by introgression of new molecular marker technology especially microsatellite analysis that facilitates the traditional breeding process [5]. In many studies a number of QTLs for agronomically important traits have been identified in wheat by microsatellite analysis including plant height (PH), heading date (HD), GYLD, tiller number per plant, ear number per unit area, ear length, spikelet number, grain number, compactness of ear and grain weight [6-12].

The purpose of the research is to find marker-trait associations in the investigated varieties. 


\section{Materials and methods}

The analyzed material consists of 47 bread winter wheat varieties (Triticum aestivum L.) originated at the Plant Breeding and Genetics Institute - National Center of Seed and Cultivar Investigations (PBGI - NCSCI) and entered into the State Register of plant varieties suitable for dissemination in Ukraine during different years: 'Hospodynia' (2007), 'Scarbnytsia' (2007), 'Kosovytsia' (2008), 'Antonivka' (2008), 'Zamozhnist' (2008), 'Blahodarka odeska' (2009), 'Misiia odeska' (2009), 'Dalnytska' (2005), 'Yednist' (2008), 'Kiriia' (2004), 'Liona' (2005), 'Kuialnyk' (2003), 'Poshana' (2004), 'Zaporuka' (2008), 'Bunchuk' (2009), 'Podiaka' (2008), 'Oksana' (2007), 'Zahrava odeska' (2010), 'Epokha odeska' (2010), 'Lytanivka' (2008), 'Sluzhnytsia odeska' (2009), 'Hoduvalnytsia odeska' (2009), 'Istyna odeska' (2010), 'Zmina' (2007), 'Dovira' (2009), 'Krasen' (2009), 'Otaman' (2008), 'Borvii' (2010), 'Turunchuk' (2008), 'Diuk' (2008), 'Nebokrai' (2011), 'Khyst' (2013), 'Pylypivka' (2011), 'Zorepad' (2011), 'Zhaivir' (2010), 'Uzhynok' (2010), 'Hurt' (2013), 'Dobrochyn' (2013), 'Vatazhok' (2011), 'Poliovyk' (2009), 'Holubka odeska' (2011), 'Kniahynia Olha' (2011), 'Lebidka odeska' (2 samples, 2011), 'Zhuravka odeska' (2011), 'Bezmezhna' (2008), 'Lastivka odeska' (2011). Such variety as 'Albatros odeskyi' (1990) and two collection samples of 'Bezosta 1' variety (1955) were taken into research as reference samples according to the recommendation of the Ukrainian Institute for Plant Variety Examination of the Ministry of Agriculture and Food of Ukraine (UIPVE of MAFU) for distinctness, uniformity and stability (DUS) testing of new varieties of bread wheat (Triticum aestivum L.) for the purpose of granting the Breeders' Right [13]. 'Albatros odeskyi' variety was created and recommended for breeding practices and production by Academician N. A. Lytvynenko as a source that ensures genetic productivity, frost resistance, drought tolerance, heat resistance and has excellent grain quality [14], so we used this variety as a reference crop in field experiments.

The mentioned genotypes of 47 bread winter wheat varieties were characterized using 17 SSR markers abundantly described in the literature $[1,5]$, the part of which was given in the guidelines on the differentiation and identification of wheat varieties [15], and additionally they were used in our previous researches [16, 17].

Genomic DNA was extracted from seedlings using modified CTAB method [18]. Polymerase chain reactions (PCR) was performed on a Tertsyk thermocycler (DNA Technology, Russia) according to Röder et al. [19], with 35 instead of 45 cycles of denaturation for $1 \mathrm{~min}$ at $94^{\circ} \mathrm{C}$, annealing for $1 \mathrm{~min}$ at $50{ }^{\circ} \mathrm{C}\left(55\right.$ or $60{ }^{\circ} \mathrm{C}$ depending on the primer) and extension for $1 \mathrm{~min}$ at $72{ }^{\circ} \mathrm{C}$ followed by a final extension step for 5 min at $72{ }^{\circ} \mathrm{C}$. PCR was carried out in a final reaction volume of $25 \mu \mathrm{L}$ containing: $60 \mathrm{ng}$ of DNA; $0.25 \mu \mathrm{M}$ of each primer; $10 \mathrm{x}$ PCR-buffer (40 mM Tris-HCl pH 8,4; $25 \mathrm{mM}$ $\mathrm{KCl} ; 1.5 \mathrm{mM}$ of $\mathrm{MgCl}_{2} ; 0,01 \%$ Tween-20); 0,2 $\mathrm{mM}$ of each dNTP; 1 unit of Taq-polymerase. The amplification products $(10-\mu \mathrm{L}$ aliquot of the PCR mixture) were separated in $7 \%$ polyacrylamide gel in $1 \times$ TBE using Höfer scientific instruments (USA). Visualization of PCR products was performed by staining of gels in $\mathrm{AgNO}_{3}$ according to "Silver sequence TM DNA Sequencing System Technical Manual".

Image Master VDS video system (Amersham Pharmacia Biotech, USA) was used to assess the fragment size of the alleles at each microsatellite locus. The pUC19 DNA/ MspI and $100 \mathrm{bp}$ DNA Ladder were used as standard ladders. Statistical processing of the results obtained was carried out by standard methods [20].

The seeds provided by the different research departments of PBGI - NCSCI have been sown on separate plots in field experiment conditions of $2010 / 11,2011 / 12$, $2012 / 13,2013 / 14$ growing years by the scientists of the laboratory of Variety Investigation and Breeding Process Modeling of PBGI located in Odesa, Ukraine ( $46^{\circ} 27^{\prime} 3$ ", $\left.30^{\circ} 39^{\prime} 18^{\prime \prime}\right) .5$ seedlings of each variety were taken in a random way for microsatellite analysis (MS-analysis), among which one was used for the further growing under ear-row scheme. Each season the reference microsatellite analysis was performed to determine the purity of seeds and prevent possible contamination. The obtained grain material was analyzed for a number of important agronomic traits: heading date (HD), plant height $(\mathrm{PH})$, awn size (AS), ear colour (EC), ear size (ES). HD was estimated during three growing years $(2010 / 11,2011 / 12,2012 / 13)$ as days since the $1^{\text {st }}$ of May when approximately half of ears in a plot have half emerged from the flag leaf. PH $(\mathrm{cm})$ is made up of a main stem length including the length of the ear and was evaluated during four growing years (2010/11, 2011/12, 2012/13, 2013/14). Awn size was determined according to the scale of 1 up to 7 as it was recommended by [13]: 1 - absolutely without awns (as 'Bezosta 1' va- 
riety), 2 - short awns, 3 - average awns, 5 long awns, 7 - very long awns. It was estimated during three growing years $(2010 / 11$, $2011 / 12,2012 / 13)$. Among the studied varieties, there was found none that would have long or very long awns. EC and ES were evaluated during two growing years (2011/12, $2012 / 13)$.

EC was determined as the degree of colour intensity with the help of the scale of 1 to 5 ( 1 - white, 3 - straw yellow, 5 - red). Among tested varieties we have not revealed any possessing smoky grey or black ears which were mentioned to exist according to [13]. For determining the ear size, a 9-point scale (1 very short, 3 - short, 5 - medium, 7 - long, 9 - very long) has been used.

'Albatros odeskyi' was included in the trial as a reference variety. It was planted once every 8 studied varieties, which were sown in two rows, for more accurate registration of the whole set of features according to the method provided by [13]. Scoring indicators for ear size, awn size and ear colour were made as it was suggested by UPOV and UIEPV of MAFU [13]. Thus, 'Albatros odeskyi' variety is characterized by 2 points for AS, 3 for $\mathrm{EC}$ and 5 for ES.

All data were evaluated by means of descriptive statistic instruments of EXCEL package. Averaged values and their errors were calculated for every group carrying allele of interest, for each season separately. Significance of between-group differences was estimated by t-criterion. For illustration of differences between alternative pairs of alleles (as shown in Figures 2 and 3), along with investigation of the structure of total variation of $\mathrm{HD}$ and $\mathrm{PH}$ traits, ANOVA method was used with alleles and years as main factors; the calculations were performed by GLM instrument from AGROBASE 21 package (Agronomix Software Inc., Canada) [21].

\section{Results and discussion}

Mean values of evaluated traits in all years analyzed showed considerable levels of intervariety diversity (Table 1). Early HD was recorded first in 'Zaporuka' variety, while 'Oksana' had the latest date of HD. The highest value of $\mathrm{PH}$ was revealed in 'Polovyk', while the smallest one 'Zaporuka' variety had. The shortest AS was in 'Zmina' variety, whereas 'Hospodynia', 'Misiia odeska', 'Kiriia', Liona', 'Kuialnyk', 'Poshana', 'Podiaka', 'Zahrava odeska', 'Dovira' varieties had average AS. Both samples of 'Lebidka odeska' variety had the smallest value of EC, while 'Krasen' variety showed the highest one (5, red). 'Oksana' variety had the smallest value of ES, whereas 'Epokha odeska' variety was characterized by the highest one among the studied varieties.

Table 1

Levels of inter-variety diversity for heading date (HD), plant height (PH), awn size (AS), ear colour (EC), ear size (ES)*

\begin{tabular}{|l|c|c|c|c|c|}
\hline \multicolumn{1}{|c|}{ Data } & HD & PH & AS & EC & ES \\
\hline Mean & 15,36 & 79,57 & 2,64 & 2,21 & 5,13 \\
SD & 1,25 & 5,82 & 0,72 & 0,74 & 0,44 \\
CV & 8,15 & 7,32 & 27,12 & 33,56 & 8,62 \\
min & 12,33 & 65,00 & 1,00 & 1,00 & 4,00 \\
max & 18,33 & 95,00 & 3,00 & 5,00 & 6,00 \\
\hline
\end{tabular}

* $\mathrm{HD}$ was estimated as days since the $1^{\text {st }}$ of May; $\mathrm{PH}$ is given in $\mathrm{cm}$; $\mathrm{AS}, \mathrm{EC}$ and $\mathrm{ES}$ are given in points evaluated by 7-point, 5-point and 9-point scales, respectively; Mean average values; SD - standard deviation; CV - coefficient of variation, $\%$.

Significant marker-trait associations for $\mathrm{HD}, \mathrm{PH}, \mathrm{AS}, \mathrm{EC}$, ES during two-four analyzed years are denoted by asterisks $(* p \leq 0.05$ and $* * \mathrm{p} \leq 0.01$ ) in Tables 2, 3 (supplementary material). The highest number of associations was revealed for $\mathrm{HD}$ and $\mathrm{PH}$. The SSR markers $X g w m 186-5 \mathrm{~A}$ and $X g w m 3-3 \mathrm{D}$ to a great degree were associated with all analyzed traits and shown stability during three years for $\mathrm{PH}$. Markers Xtaglgap-1B, Xgwm155-3A showed stable associations with $\mathrm{HD}, \mathrm{PH}, \mathrm{AS}$ during all years analyzed and significant associations with EC.

Three traits were considerably associated with three markers (Xgwm408-5B, Xgwm325-6D, $X g w m 577-7 B)$, namely $X g w m 408-5 B$ showed association for HD, PH and EC; Xgwm325-6D with HD, PH and AS; marker Xgwm577-7B with HD, EC and ES. Seven SSR markers were associated with two studied traits, whereas three markers showed associations with only one trait, namely for HD (Xgwm389-3B), AS (Xgwm190-5D), ES (Xgwm18-1B).

Table 2

The number of alleles according to their associations with heading date (HD), plant height (PH), awn size (AS), ear colour (EC), ear size (ES)

\begin{tabular}{|l|c|c|c|c|c|}
\hline \multicolumn{1}{|c|}{ Characteristics } & $\mathrm{HD}^{*}$ & $\mathrm{PH}$ & $\mathrm{AS}$ & $\mathrm{EC} *$ & $\mathrm{ES}$ \\
\hline $\begin{array}{l}\text { Number of alleles increasing } \\
\text { the value of a studied trait }\end{array}$ & 20 & 17 & 15 & 10 & 3 \\
$\begin{array}{l}\text { Number of alleles decreasing } \\
\text { the value of a studied trait }\end{array}$ & 15 & 22 & 17 & 10 & 5 \\
$\begin{array}{l}\text { Alternate } \\
\text { Rest }\end{array}$ & 5 & 2 & 1 & 3 & 1 \\
\hline
\end{tabular}

* Increasing values mean the earlier date of HD;

** Increasing values mean the lighter EC. 


\section{Supplementary material}

Table 3

Significant associations of SSR alleles with heading date (HD), plant height (PH), awn size (AS), ear colour (EC), ear size (ES), represented as signs, revealed in field experiments during 2-4 years

(the years of harvesting are shown in the header)

\begin{tabular}{|c|c|c|c|c|c|c|c|c|c|c|c|c|c|c|c|}
\hline \multirow{2}{*}{ Locus } & \multirow{2}{*}{ Allele } & \multicolumn{3}{|c|}{ HD } & \multicolumn{4}{|c|}{$\mathrm{PH}$} & \multicolumn{3}{|c|}{ AS } & \multicolumn{2}{|c|}{$\mathrm{EC}$} & \multicolumn{2}{|c|}{ ES } \\
\hline & & 2011 & 2012 & 2013 & 2011 & 2012 & 2013 & 2014 & 2011 & 2012 & 2013 & 2012 & 2013 & 2012 & 2013 \\
\hline \multirow{7}{*}{$\begin{array}{l}\text { Xgwm357 } \\
(1 \mathrm{~A})\end{array}$} & 116 & $\downarrow * *$ & $\downarrow *$ & $\downarrow * *$ & na & $\downarrow *$ & & & na & $\uparrow * *$ & $\uparrow *$ & $\uparrow *$ & & & $\uparrow *$ \\
\hline & 119 & & & & & & & & & & & & & & \\
\hline & 121 & & & & & & & & & & & & & & \\
\hline & 123 & $\uparrow * *$ & $\uparrow *$ & 个** & & $\uparrow *$ & & & & $\downarrow *$ & $\downarrow^{*}$ & & & & $\downarrow * *$ \\
\hline & 125 & & $\uparrow * *$ & $\uparrow * *$ & & & & & & $\uparrow * *$ & $\uparrow * *$ & $\uparrow * *$ & & & $\uparrow * *$ \\
\hline & 128 & $\uparrow *$ & $\uparrow *$ & $\uparrow * *$ & & $\uparrow *$ & & & & & & $\uparrow * *$ & & & $\uparrow * *$ \\
\hline & 134 & & $\uparrow *$ & 个** & & $\uparrow *$ & & & & $\downarrow * *$ & $\downarrow * *$ & $\downarrow * *$ & & & \\
\hline \multirow{4}{*}{$\begin{array}{l}\text { Xgwm18 } \\
\text { (1B) }\end{array}$} & 186 & & & & & & & & & & & & & & $\downarrow *$ \\
\hline & 188 & & & & & & & & & & & & & & \\
\hline & 192 & & & & & & & & & & & & & $\uparrow *$ & $\uparrow *$ \\
\hline & 196 & & & & & & & & & & & & $\uparrow *$ & $\downarrow *$ & $\downarrow *$ \\
\hline \multirow{5}{*}{$\begin{array}{l}\text { Taglgap } \\
\text { (1B) }\end{array}$} & 215 & $\uparrow * *$ & $\uparrow * *$ & $\uparrow * *$ & $\uparrow *$ & & & $\downarrow *$ & $\uparrow * *$ & $\uparrow * *$ & $\uparrow * *$ & $\downarrow * *$ & $\downarrow^{*}$ & 个* & \\
\hline & 218 & $\downarrow * *$ & $\downarrow_{* *}$ & $\downarrow * *$ & $\downarrow *$ & $\downarrow_{*}$ & $\downarrow *$ & $\downarrow_{* *}$ & $\uparrow * *$ & $\uparrow * *$ & $\uparrow * *$ & $\uparrow * *$ & $\uparrow *$ & $\downarrow *$ & \\
\hline & 235 & & & $\downarrow *$ & & & & & & & & & & & \\
\hline & 238 & $\uparrow * *$ & $\uparrow * *$ & 个** & $\uparrow *$ & $\uparrow *$ & 个* & $\uparrow * *$ & $\downarrow * *$ & $\downarrow * *$ & $\downarrow * *$ & & & & \\
\hline & 265 & & & & & & & & & & & & & & \\
\hline \multirow{5}{*}{$\begin{array}{l}\text { Xgwm095 } \\
(2 \mathrm{~A})\end{array}$} & 110 & na & na & $\uparrow * *$ & & & & $\downarrow_{*}$ & & & & & & & \\
\hline & 120 & & & $\downarrow_{* *}$ & $\downarrow *$ & $\downarrow_{* * *}$ & $\downarrow *$ & $\downarrow * *$ & & $\downarrow *$ & $\downarrow_{*}$ & & & $\downarrow_{*}$ & \\
\hline & 122 & & & & $\uparrow *$ & $\uparrow * *$ & $\uparrow *$ & $\uparrow * *$ & & $\downarrow *$ & $\downarrow_{*}$ & & & $\uparrow *$ & \\
\hline & 128 & & $\uparrow *$ & & & & & & & $\uparrow *$ & $\uparrow *$ & & & & \\
\hline & 130 & & $\downarrow *$ & $\downarrow * *$ & na & $\downarrow * *$ & $\downarrow *$ & $\downarrow *$ & & & & & & & \\
\hline \multirow{6}{*}{$\begin{array}{l}\text { Xgwm3 } \\
(2 \mathrm{D})\end{array}$} & 75 & & & & & & & & & & & & & & \\
\hline & 77 & & 个* & & & $\uparrow * *$ & $\uparrow *$ & & & $\downarrow_{* *}$ & $\downarrow * *$ & $\downarrow *$ & $\downarrow *$ & $\downarrow_{*}$ & $\uparrow *$ \\
\hline & 79 & $\uparrow *$ & & & $\uparrow *$ & & & & $\downarrow * *$ & $\downarrow * *$ & $\downarrow * *$ & $\downarrow *$ & & $\uparrow *$ & \\
\hline & 81 & & $\downarrow *$ & & & $\uparrow *$ & $\uparrow *$ & & & & & $\uparrow *$ & $\uparrow *$ & $\downarrow *$ & \\
\hline & 83 & $\uparrow *$ & $\uparrow * *$ & & & & & & na & $\uparrow *$ & $\uparrow *$ & $\uparrow *$ & $\uparrow *$ & & $\downarrow *$ \\
\hline & 86 & $\uparrow * *$ & $\uparrow *$ & & & $\downarrow * *$ & $\downarrow *$ & & $\downarrow *$ & & $\uparrow *$ & $\uparrow * *$ & $\uparrow *$ & $\downarrow$ 政 & \\
\hline $\begin{array}{l}\text { Xgwm3 } \\
(2 \mathrm{D})\end{array}$ & 88 & $\downarrow$ ** & $\downarrow * *$ & & $\downarrow *$ & $\downarrow *$ & $\downarrow *$ & & $\uparrow * *$ & $\uparrow * *$ & $\uparrow * *$ & 个** & $\uparrow * *$ & $\downarrow * *$ & $\downarrow *$ \\
\hline \multirow{10}{*}{$\begin{array}{l}\text { Xgwm155 } \\
(3 \mathrm{~A})\end{array}$} & 129 & na & na & $\downarrow *$ & & & & & & & & & & & \\
\hline & 135 & & & & & & & & & & & & & & \\
\hline & 137 & na & na & $\downarrow *$ & na & na & na & $\downarrow *$ & & & & & & & \\
\hline & 139 & & $\uparrow *$ & & $\downarrow *$ & $\downarrow *$ & & & $\uparrow *$ & $\uparrow * *$ & $\uparrow * *$ & 个** & $\uparrow *$ & $\downarrow_{*}$ & \\
\hline & 141 & $\uparrow *$ & & $\uparrow * *$ & 个* & $\uparrow *$ & $\uparrow * *$ & $\uparrow * *$ & $\downarrow *$ & $\downarrow * *$ & $\downarrow * *$ & $\downarrow * *$ & & $\uparrow *$ & \\
\hline & 143 & $\downarrow *$ & $\downarrow *$ & $\downarrow * *$ & $\downarrow *$ & & & & $\uparrow * *$ & $\uparrow * *$ & $\uparrow * *$ & $\uparrow * *$ & $\downarrow *$ & & \\
\hline & 145 & & & & $\downarrow *$ & & & $\downarrow_{*}$ & & $\uparrow * *$ & $\uparrow * *$ & $\downarrow *$ & $\downarrow *$ & $\downarrow_{*}$ & \\
\hline & 147 & & $\uparrow *$ & $\downarrow$ 政 & $\downarrow *$ & $\downarrow *$ & $\downarrow * *$ & $\downarrow$ 政 & $\uparrow * *$ & $\uparrow * *$ & $\uparrow * *$ & $\downarrow *$ & $\downarrow *$ & $\uparrow * *$ & \\
\hline & 149 & $\uparrow *$ & & $\uparrow *$ & $\downarrow *$ & $\downarrow *$ & $\downarrow *$ & $\downarrow * *$ & $\uparrow * *$ & $\uparrow * *$ & $\uparrow * *$ & $\downarrow * *$ & $\downarrow * *$ & $\uparrow * *$ & \\
\hline & 152 & & $\downarrow *$ & $\downarrow *$ & na & & $\downarrow *$ & $\downarrow_{* *}$ & na & $\uparrow * *$ & $\uparrow * *$ & 个** & & $\downarrow_{* *}$ & \\
\hline \multirow{4}{*}{$\begin{array}{l}\text { Xgwm389 } \\
(3 \mathrm{~B})\end{array}$} & 117 & & & $\uparrow * *$ & & $\uparrow *$ & & $\uparrow+$ & & $\downarrow *$ & & & & $\downarrow_{-}$ & \\
\hline & 119 & & $\uparrow *$ & $\uparrow * *$ & & & & & & & & & $\downarrow *$ & $\downarrow_{*}$ & \\
\hline & 134 & & $\uparrow *$ & $\uparrow *$ & & & & & & & & & & $\downarrow_{*}$ & \\
\hline & 136 & & & $\downarrow * *$ & & $\downarrow *$ & & $\downarrow_{+}$ & & & & & $\uparrow *$ & $\downarrow_{*}$ & \\
\hline \multirow{3}{*}{$\begin{array}{l}\text { Xgwm389 } \\
\text { (3B) }\end{array}$} & 138 & & & $\uparrow * *$ & & & & & & & & & $\uparrow *$ & & \\
\hline & 142 & & & $\uparrow *$ & na & $\uparrow *$ & & & na & $\uparrow *$ & $\uparrow_{+}$ & & & $\uparrow *$ & \\
\hline & 145 & & $\downarrow *$ & & & & & & & & & & & & \\
\hline & 185 & & & & & & & & & & & & $\uparrow *$ & & \\
\hline & 189 & na & na & $\downarrow_{*}$ & & & & $\downarrow *$ & & & & & & & \\
\hline Xgwm165/1 & 191 & & $\downarrow *$ & $\uparrow *$ & & $\uparrow * *$ & & $\uparrow * *$ & & & & & $\downarrow * *$ & $\uparrow *$ & \\
\hline & 193 & & $\downarrow *$ & $\downarrow * *$ & & $\downarrow * *$ & & $\downarrow$ ** & & & & & $\uparrow * *$ & $\downarrow_{*}$ & \\
\hline & 195 & & $\uparrow *$ & 个** & & $\uparrow * *$ & & $\uparrow * *$ & & & & & & $\uparrow *$ & \\
\hline
\end{tabular}




\begin{tabular}{|c|c|c|c|c|c|c|c|c|c|c|c|c|c|c|c|}
\hline \multirow{2}{*}{ Locus } & \multirow{2}{*}{ Allele } & \multicolumn{3}{|c|}{ HD } & \multicolumn{4}{|c|}{$\mathrm{PH}$} & \multicolumn{3}{|c|}{ AS } & \multicolumn{2}{|c|}{ EC } & \multicolumn{2}{|c|}{ ES } \\
\hline & & 2011 & 2012 & 2013 & 2011 & 2012 & 2013 & 2014 & 2011 & 2012 & 2013 & 2012 & 2013 & 2012 & 2013 \\
\hline \multirow{8}{*}{$\begin{array}{l}\text { Xgwm186 } \\
(5 \mathrm{~A})\end{array}$} & 102 & & & & & 个** & $\uparrow * *$ & $\uparrow * *$ & & & & & & $\uparrow * *$ & $\uparrow * *$ \\
\hline & 107 & & $\uparrow *$ & $\uparrow *$ & na & $\uparrow * *$ & & $\uparrow *$ & na & $\downarrow *$ & $\downarrow *$ & $\downarrow_{* *}$ & $\downarrow *$ & $\uparrow * *$ & \\
\hline & 113 & & & & & $\downarrow_{* *}$ & $\downarrow *$ & $\uparrow * *$ & & & & & & $\uparrow * *$ & \\
\hline & 115 & & & $\uparrow * *$ & & & $\downarrow * *$ & & & & & $\uparrow * *$ & & $\downarrow * *$ & $\downarrow * *$ \\
\hline & 125 & & & $\downarrow * *$ & & $\downarrow * *$ & $\downarrow *$ & $\downarrow * *$ & & $\uparrow *$ & $\uparrow *$ & $\uparrow * *$ & $\uparrow *$ & $\uparrow * *$ & $\uparrow+$ \\
\hline & 129 & & $\downarrow *$ & $\downarrow *$ & & $\downarrow * *$ & & $\downarrow *$ & & & & $* *$ & $*$ & 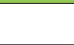 & \\
\hline & 135 & & & & & $\downarrow * *$ & $\downarrow * *$ & $\downarrow *$ & & & & $\downarrow_{*}$ & & & \\
\hline & 139 & & & $\uparrow * *$ & na & . & & & na & $\downarrow *$ & $\downarrow *$ & & $\downarrow *$ & $\downarrow *$ & \\
\hline $\begin{array}{l}\text { Xgwm186 } \\
(5 \mathrm{~A})\end{array}$ & 142 & & & $\downarrow *$ & & $\uparrow *$ & 个* & $\uparrow *$ & & & & $\downarrow *$ & $\downarrow *$ & $\uparrow *$ & \\
\hline \multirow{6}{*}{$\begin{array}{l}\text { Xgwm } 408 \\
(5 \mathrm{~B})\end{array}$} & 148 & & & & & & & & & & & & & & \\
\hline & 162 & & & & na & na & na & $\downarrow *$ & & & & & & & \\
\hline & 178 & & & & & & & & & & & & & & $\uparrow *$ \\
\hline & 185 & & & & & & & & & & & & & & \\
\hline & 188 & & $\downarrow * *$ & $\downarrow * *$ & & $\downarrow * *$ & $\downarrow *$ & $\downarrow * *$ & & & & $\uparrow * *$ & $\uparrow *$ & & \\
\hline & 192 & & $\uparrow * *$ & $\uparrow * *$ & & $\uparrow * *$ & $\uparrow *$ & $\uparrow * *$ & & & $\downarrow_{*}$ & $\downarrow_{* * *}$ & $\downarrow *$ & & $\downarrow *$ \\
\hline \multirow{3}{*}{$\begin{array}{l}\text { Xgwm190 } \\
\text { (5D) }\end{array}$} & 204 & & & & & & & & & 个** & $\uparrow * *$ & & & & \\
\hline & 208 & & & & & & & & & $\downarrow * *$ & $\downarrow * *$ & & & & \\
\hline & 210 & & & & & & & & & & & & & & \\
\hline \multirow{5}{*}{$\begin{array}{l}\text { Xgwm325 } \\
\text { (6D) }\end{array}$} & 115 & $\downarrow * *$ & $\downarrow * *$ & $\downarrow_{* *}$ & na & $\downarrow * *$ & $\downarrow *$ & $\downarrow * *$ & na & & $\uparrow * *$ & 个** & & & \\
\hline & 120 & na & $\downarrow * *$ & $\downarrow *$ & na & $\downarrow * *$ & $\downarrow * *$ & & na & & $\uparrow * *$ & $\uparrow * *$ & & & \\
\hline & 128 & $\uparrow *$ & $\uparrow * *$ & $\uparrow * *$ & & & & & na & & $\uparrow * *$ & $\uparrow * *$ & & & \\
\hline & 134 & na & na & $\uparrow *$ & na & na & na & $\downarrow *$ & & & & & & & \\
\hline & 138 & na & $\downarrow * *$ & & na & $\downarrow *$ & $\downarrow *$ & & na & & $\uparrow * *$ & $\uparrow * *$ & & & \\
\hline \multirow{5}{*}{$\begin{array}{l}\text { Xgwm325 } \\
(6 \mathrm{D})\end{array}$} & 142 & & & & & & & & & & & & & & \\
\hline & 144 & $\downarrow * *$ & $\downarrow *$ & $\uparrow *$ & & $\downarrow *$ & & & $\uparrow * *$ & 个** & $\uparrow * *$ & $\uparrow * *$ & & & \\
\hline & 146 & $\downarrow_{* *}$ & $\downarrow * *$ & $\downarrow *$ & & & & $\downarrow *$ & & & $\uparrow * *$ & $\uparrow * *$ & & & \\
\hline & 148 & $\uparrow * *$ & 个** & $\uparrow *$ & & & & $\uparrow * *$ & $\downarrow * *$ & $\downarrow * *$ & $\downarrow * *$ & $\downarrow_{*}$ & & & \\
\hline & 150 & $\uparrow * *$ & $\uparrow * *$ & $\uparrow * *$ & na & $\uparrow * *$ & $\uparrow * *$ & $\uparrow * *$ & na & $\downarrow * *$ & $\downarrow * *$ & $\downarrow_{* * *}$ & & & \\
\hline \multirow{5}{*}{$\begin{array}{l}\text { Xgwm577 } \\
\text { (7B) }\end{array}$} & 137 & $\uparrow *$ & & $\uparrow *$ & & & & & & & & $\downarrow_{* * *}$ & $\downarrow * *$ & $\uparrow * *$ & \\
\hline & 152 & & & & & & & & & & & $\uparrow * *$ & $\uparrow * *$ & & \\
\hline & 171 & $\uparrow *$ & & $\downarrow *$ & & & & & & & & $\uparrow_{+}$ & $\uparrow * *$ & $\downarrow * *$ & $\downarrow *$ \\
\hline & 173 & & & $\uparrow *$ & & & & & & & & & & $\downarrow * *$ & $\downarrow * *$ \\
\hline & 175 & $\downarrow *$ & & $\downarrow *$ & & & & & & & & $\uparrow * *$ & $\downarrow *$ & $\uparrow *$ & $\uparrow * *$ \\
\hline & 105 & & & & & & & & & & & & & & \\
\hline Xqwm437 & 107 & & $\downarrow_{*}$ & & & $\downarrow *$ & $\downarrow *$ & & $\uparrow * *$ & 个** & $\uparrow * *$ & & & & \\
\hline (7D) & 109 & & $\uparrow *$ & & & $\uparrow *$ & $\uparrow *$ & & $\downarrow * *$ & $\downarrow * *$ & $\downarrow * *$ & & & & \\
\hline & 113 & & $\downarrow *$ & & & & & & & & & & & & \\
\hline Xgwm44 & 176 & & & $\downarrow * *$ & & & & & & & & $\uparrow *$ & & $\downarrow_{*}$ & \\
\hline (7D) & 178 & & & & & & & & & & & & & & \\
\hline & 180 & & & & $\downarrow *$ & & & & & & & $\uparrow *$ & & $\downarrow *$ & \\
\hline Xgwm44 & 183 & & & $\downarrow_{*}$ & & $\uparrow * *$ & & & & & & $\downarrow_{*}$ & $\downarrow *$ & $\uparrow *$ & \\
\hline (7D) & 185 & & & $\uparrow * *$ & $\uparrow *$ & $\uparrow * *$ & & & & & & & & $\downarrow *$ & \\
\hline & 187 & & & & na & $\downarrow * *$ & & & & & & $\downarrow_{*}$ & $\uparrow *$ & & \\
\hline & 138 & & & $\uparrow *$ & na & $\uparrow * *$ & & $\uparrow * *$ & & & & & & & \\
\hline & 142 & & & & & & & & & & & & & & \\
\hline & 146 & na & na & $\downarrow * *$ & & & & & & & & & & & \\
\hline & 152 & na & & $\downarrow * *$ & na & na & na & $\downarrow_{* *}$ & & & & & & & \\
\hline Barc126 & 156 & & & $\uparrow * *$ & & $\downarrow * *$ & & $\downarrow *$ & $\uparrow+$ & $\uparrow * *$ & $\uparrow * *$ & & & & $\uparrow * *$ \\
\hline (7D) & 158 & & & & & & & & & & & & & & \\
\hline & 160 & & & & & & & & & & & & & & \\
\hline & 162 & na & & $\downarrow$ $* *$ & & & & $\downarrow_{* *}$ & & & & & & & \\
\hline & 164 & & & $\uparrow * *$ & & $\downarrow * *$ & & $\uparrow * *$ & $\downarrow_{-}$ & $\downarrow * *$ & $\downarrow * *$ & & & & $\downarrow * *$ \\
\hline & 166 & & & $\uparrow * *$ & & 个** & & $\uparrow * *$ & & & & & & & \\
\hline Wmc405 & 210 & & & & & & & & & & & & & & \\
\hline (7D) & 212 & & & & & & & & & & & & & & \\
\hline
\end{tabular}




\begin{tabular}{|c|c|c|c|c|c|c|c|c|c|c|c|c|c|c|c|}
\hline \multirow{2}{*}{ Locus } & \multirow{2}{*}{ Allele } & \multicolumn{3}{|c|}{ HD } & \multicolumn{4}{|c|}{ PH } & \multicolumn{3}{|c|}{ AS } & \multicolumn{2}{|c|}{$\mathrm{EC}$} & \multicolumn{2}{|c|}{ ES } \\
\hline & & 2011 & 2012 & 2013 & 2011 & 2012 & 2013 & 2014 & 2011 & 2012 & 2013 & 2012 & 2013 & 2012 & 2013 \\
\hline \multirow{4}{*}{$\begin{array}{l}\text { Wmc405 } \\
\text { (7D) }\end{array}$} & 216 & na & $\downarrow *$ & & & & & & & & & $\uparrow *$ & & & \\
\hline & 218 & & $\uparrow *$ & & & $\downarrow * *$ & $\downarrow *$ & & $\uparrow *$ & & & $\downarrow *$ & & & \\
\hline & 220 & $\uparrow *$ & $\downarrow *$ & $\downarrow *$ & & $\downarrow *$ & $\downarrow *$ & & & & & & & & \\
\hline & 222 & $\uparrow *$ & $\uparrow *$ & $\uparrow *$ & & $\uparrow * *$ & $\uparrow *$ & & $\downarrow *$ & & & $\uparrow *$ & & & \\
\hline
\end{tabular}

* Significant at $\mathrm{p} \leq 0.05$.

* * Significant at $p \leq 0.01 ; \uparrow(+)$ and $\downarrow(-)$ symbols indicate increasing or reducing, respectively, of the studied values depending on alleles associated with these values; shading area reveals the most significant and stable marker-trait associations; na - not available.

For HD 13, marker-trait associations (MTA) were shown to be stable during three growing years, especially alleles Xgwm $357_{123}$, Xgwm $357_{128}$, Xtaglgap $_{215}$ (Fig. 1), Xtaglgap ${ }_{238}$ (Fig. 1), Xgwm 325 $5_{128}$, Xgwm325 148, Xgwm $325_{150}$ and Xwmc405 ${ }_{222}$ were found to be significantly associated with the later date of HD while alleles Xgwm $357_{116}$, Xtaglgap ${ }_{218}, X g w m 155_{143}$,
Xgwm $325_{115}, X g w m 325_{146}$ were strongly associated with the earlier date of HD. Additionally, during two growing years 12 alleles were significantly associated with the later date of HD while 10 alleles showed association with the earlier date of HD. The HD values associated with presence of contrast alleles pairs in seven different loci are shown in Fig. 2.

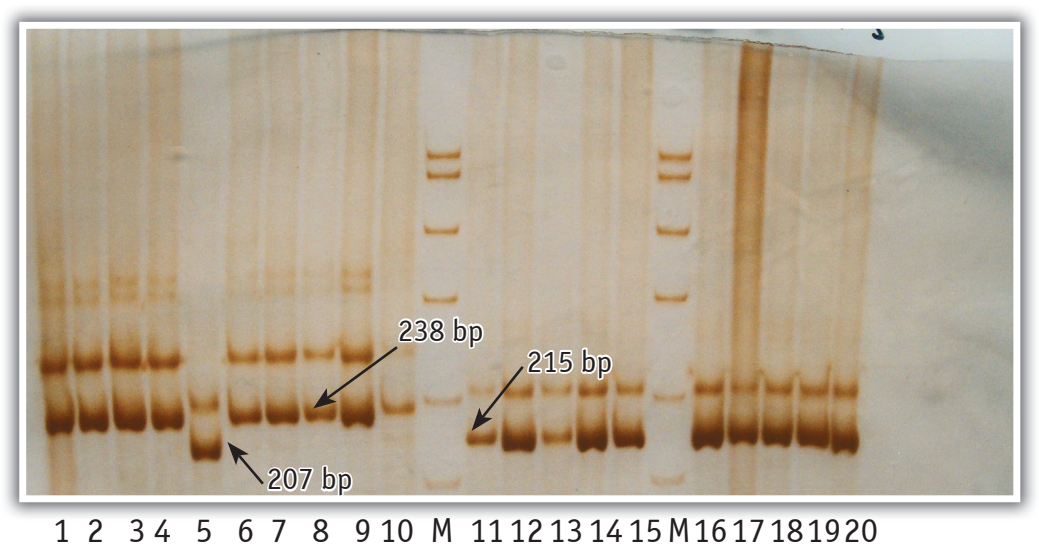

Fig. 1. Electrophoregram of DNA amplification products by the Xtaglgap-1B locus of 'Dalnytska' (1-5), 'Yednist' (6-10), 'Bezosta 1' (11-15), 'Oksana' (16-20) varieties.

(M - molecular weight marker pUC 19/Msp I. The arrows indicate sizes in bp of fragment amplification)

The largest quantity of MTAs (39) during several growing years was revealed for $\mathrm{PH} .17$ MTA were found to be stable in three-four growing years, and 22 MTA were proved to be significant in two growing years. Overall 17 SSR markers were associated with the higher value of $\mathrm{PH}$, while 22 alleles were associated with its smaller value. Fig. 3 shows $\mathrm{PH}$ values associated with presenting of contrast alleles pairs in seven different loci.

33 MTAs for AS were found to be stable and significant during two-three growing years. The shorter value of AS was greatly associated with alleles Xtaglgap ${ }_{238}, X g w m 3_{79}, X g w m 155_{141}$, Xgwm 325 ${ }_{148}, \quad X g w m 437_{109}, X$ X $\mathrm{gwm} 357_{123}$, Xgwm $357_{134}^{148}, \quad X g w m 095_{120}^{109}, \quad$ Xgwm095 ${ }_{122}^{123}$, Xgwm $3_{77}, \quad X g w m 186_{107}, \quad X g w m 186_{139}^{122}$, Xgwm190 $208, \quad X g w m 325_{150}, \quad B a r c 126_{164}^{139}$, while the larger value of AS showed associations with alleles Xtaglgap ${ }_{218}, \quad X g w m 3_{88}$, Xgwm155 ${ }_{139}, \quad X g w m 155_{143}, \quad X g w m 155_{147}$,
$X g w m 155_{149}, \quad X g w m 325_{144}, \quad X^{\prime}$ gwm $437_{107}$, Xgwm 357 ${ }_{116}, \quad X g w m 357_{125}^{14}, \quad X g w m 095_{128}$, Xgwm $3_{83}, \quad X g w m 155_{145}, \quad X g w m 155_{152}$, $X g w m 186_{125}, \quad X g w m 190_{204}, \quad B a r c 126_{156}$. Allele Xtaglgap ${ }_{215}$ appeared to be significantly associated with both smaller and higher values of AS during three growing seasons.

20 MTAs for EC and 8 MTAs for ES were proved to be significant in two growing years. The analysis revealed that 10 alleles were associated with the darker EC and 10 alleles with the lighter EC; 5 alleles were associated with the smaller value of ES while alleles Xgwm18 ${ }_{192}$, $X g w m 186_{102}, X g w m 577_{175}$ were significantly associated with the higher value of ES.

Analysis of variance (ANOVA) for HD values associated with 6 alleles in Xgwm325-6D locus and also for $\mathrm{PH}$ values associated with 5 alleles in Xgwm325-6D locus revealed significant affection on HD and $\mathrm{PH}$, respectively, despite the fact that total variation was most- 
ly attributed (88-95\% of the total sum of squares) to the years.

Thus, we have detected that marker Xtaglgap-1B showed stable association with HD, $\mathrm{PH}, \mathrm{AS}$ during all years analyzed and significant associations with EC. In studies carried out by Landjeva et al. [22] it was noted that the presence of allele Xtaglgap ${ }_{244}$ was strictly correlated with the red colour of the ear. Our investigation proved allele Xtaglgap ${ }_{215}$ to be largely associated with the darker EC while allele Xtaglgap ${ }_{218}$ to a great extent was associated with the lighter EC. In general, it was shown that the dominant gene $R g 1$, which

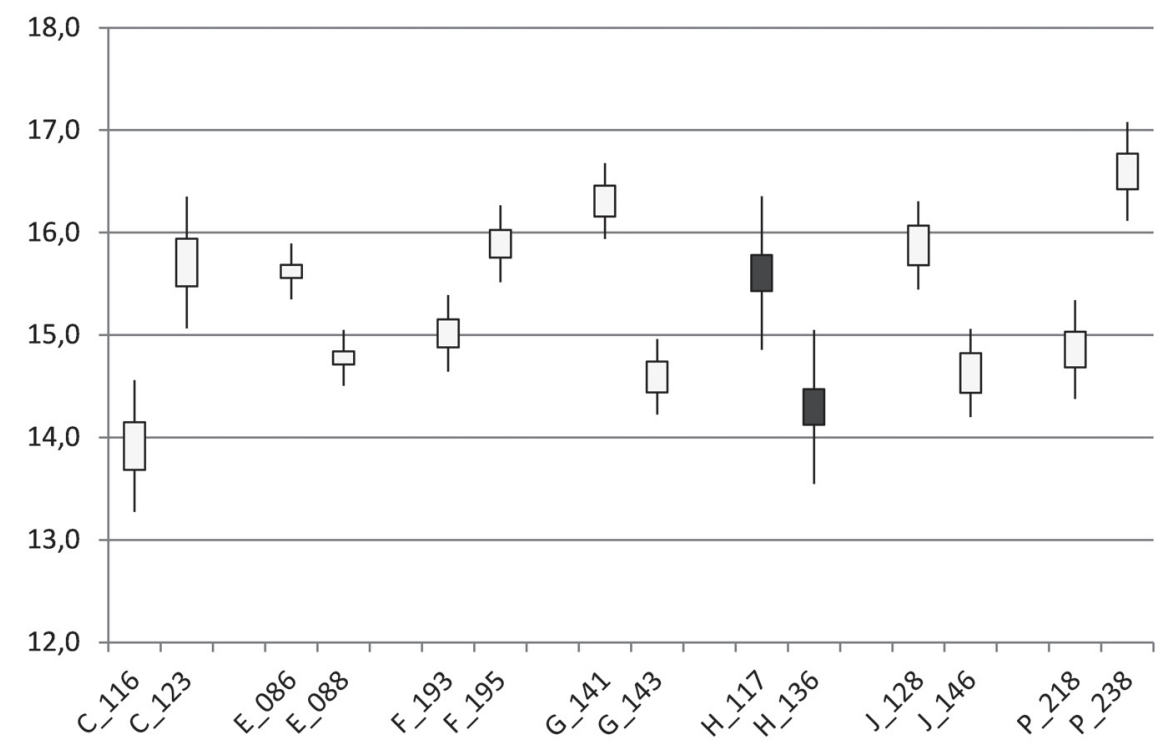

Fig. 2. HD values associated with presence of contrast alleles pairs in seven different loci, averaged values for 3 years; $Y$-axis shows days since the $1^{\text {st }}$ of May when approximately half of ears in a plot have half emerged from the flag leaf; $X$-axis shows alleles - fragment pairs (bp) in seven different loci: C - Xgwm357-1A; E - Xgwm3-3D;

F - Xgwm165/1-4A; G - Xgwm155-3A; H - Xgwm389-3B; J - Xgwm325-6D; P - Xtaglgap-1B. Bars span range Mean $\pm(\mathrm{Sd} / 2)$ and whiskers - range Mean $\pm\left(\mathrm{LSD}_{05} / 2\right)$. Solid black bars indicate pair with inter-allelic differences to be stable over the years, but with certainty below $p \leq 0.05$.

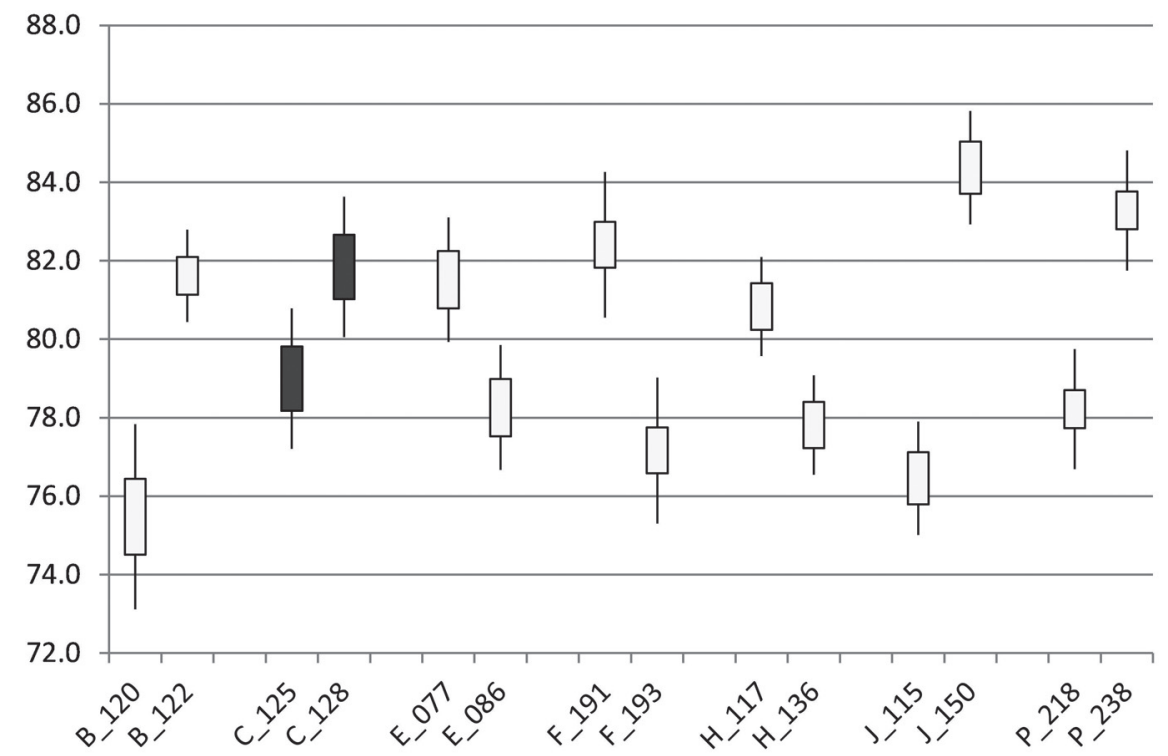

Fig. 3. PH values associated with contrast alleles pairs in seven different loci, averaged values for 4 years; $\mathrm{Y}$-axis shows $\mathrm{PH}(\mathrm{cm})$ given as a main stems length including the length of the ear; $\mathrm{X}$-axis shows alleles fragment pairs (bp) in seven different loci: B - Xgwm095-2A; C - Xgwm357-1A; E - Xgwm3-3D;

F - Xgwm165/1-4A; H - Xgwm389-3B; J - Xgwm325-6D; P - Xtaglgap-1B. Bars span range Mean $\pm(\mathrm{Sd} / 2)$ and whiskers - range Mean $\pm\left(L D_{05} / 2\right)$. Solid black bars indicate pair with inter-allelic differences to be stable over the years, but with certainty below $\mathrm{p} \leq 0.05$ 
determines the red colour of glumes is closely linked with Gli-B1 region on chromosome 1BS [23, 24]. We detected that the SSR marker Xgwm408-5B showed significant associations with HD, PH and EC. According to Leonova et al. [25], the SSR marker Xgwm408-5B appeared to be in close linkage to the vernalization response gene $V r n-B 1$ on $5 \mathrm{BL}$. In our study the SSR marker Xgwm577-7B showed significant associations with HD, EC and ES. It was previously established that the SSR marker Xgwm577-7B is closely associated with powdery mildew resistance and the $S t b 8$ resistance gene. Two loci on $1 \mathrm{~B}$ chromosome near Xgwm 18 were found to be closely linked to a $Y r$ (yellow rust) gene which was placed $1.9 \mathrm{cM}$ distally to these SSR markers. In our study allele Xgwm $18_{196}$ proved to be strongly associated with the smaller value of ES while allele $X g w m 18_{192}$ was considerably associated with the higher value of ES. Brbaklic et al. [5] identified that the QTL located near microsatellite marker $\mathrm{Xg}$ $w m 18-1 \mathrm{~B}$ explained about $23 \%$ of the total variability for $\mathrm{PH}$ in three growing years. The indicated marker showed association with HD and flowering date for two years. In our study, the same SSR marker did not have any association with HD or PH in tested varieties under climatic conditions of Southern Ukraine. Zanke et al. [12] reported the detection of 34 SSR alleles located on different wheat chromosomes which were associated with PH. 16 SSR alleles were associated with reducing the $\mathrm{PH}$ while 18 SSR alleles were associated with its enhancing. We have detected 13 SSR that were significantly associated with increasing $\mathrm{PH}$ while 16 SSR alleles - with its reducing. Additionally, 2 SSR alleles showed alternative effect on the value of PH. Zanke et al. [12] identified that allele $X g w m 357_{128}$ was significantly associatiated with $\mathrm{PH}$ and proved to be one of the most $\mathrm{PH}$ reducing alleles. In our study we have found that SSR allele Xgwm $357_{128}$ was proved to be significantly associated with the later date of HD in three growing years under Ukrainian climatic conditions. It was pointed out by Chaturvedi et al. [26] that there is the direct positive effects of $\mathrm{PH}$ on grain yield, so direct identification of genomic regions which affect expression of $\mathrm{PH}$ is important for the breeding process. In our study, 12 SSR markers associated with $\mathrm{PH}$ were determined on chromosomes 2A, 3A, $5 \mathrm{~A}, 1 \mathrm{~B}, 3 \mathrm{D}, 4 \mathrm{D}, 6 \mathrm{D}$ and $7 \mathrm{D}$. According to our investigations we have not been analyzing reduced plant height $(R h t)$ genes that have a significant effect on plant height. Confirmation of marker-trait associations in our material opens new opportunities for using the mentioned microsatellite markers for MAS.

\section{Conclusions}

In order to facilitate and improve breeding process, extensive studies, focused on finding traits of interest associated with microsatellite markers and different genomic regions of wheat, are required and could be assisted by molecular marker technologies. In conclusion, 35 marker-trait associations (MTA) for heading date, 39 for plant height, 33 for awn size, 20 for ear colour and 8 for size of ears were proved to be stable and significant during two-four different growing years. 20 SSR alleles were found to be significantly associated with the later date of HD while 15 SSR alleles - with its earlier date. 5 SSR alleles showed significant alternative effect on the value of HT. 17 SSR alleles for PH, 15 for AS and 3 for ES were found to be significantly associated with the increase of these values, respectively, while 22 SSR alleles for $\mathrm{PH}, 17$ for AS and 5 for ES were associated with their reduction. As for EC, 10 SSR alleles were found to be significantly associated with the lighter EC and 10 SSR alleles - with the darker EC; 3 SSR alleles showed alternative effect on the value of EC.

\section{Acknowledgements}

We greatly appreciate V. M. Tsevma for his generous help with currying out of field experiments and for his assistance in the data processing procedures.

\section{Bibliography}

1. Röder M. S. Fine mapping of the region on wheat chromosome 7D controlling grain weight/M. S. Röder, X.-Q. Huang, A. Börner // Funct Integr Genomics. - 2008. - Vol. 8, Iss. 1. - P. 79-86. doi: 10.1007/s10142-007-0053-8

2. Gupta P. K. Genetic and molecular basis of grain size and grain number and its relevance to grain productivity in higher plants / P. K. Gupta, S. Rustgi, N. Kumar // Genome. - 2006. - Vol. 49, No. 6. - P. 565-571. doi: 10.1139/g06-063

3. High-density mapping and comparative analysis of agronomically important traits on wheat chromosome 3A / M. Dilbirligi, M. Eraymana, B. T. Campbell [et al.] // Genomics. - 2006. Vol. 88, No. 1. - P. 74-87. doi: 10.1016/j.ygeno.2006.02.001

4. Litvinenko N. A. Breeding intensive winter bread wheat varieties for Southern Ukraine / N. A. Litvinenko // Euphytica. - 1998. - Vol. 100, No. 1-3. - P. 7-14. doi: 10.1023/A:1018383317939

5. Detection of QTLs for important agronomical traits in hexaploid wheat using association analysis / L. Brbaklic, D. Trkulja, A. Kondic-Spika [et al.] // Czech J. Genet. Plant Breed. - 2013. - Vol. 49, No. 1. - P. 1-8.

6. Mapping of quantitative trait loci determining agronomic important characters in hexaploid wheat (Triticum aestivum L.) / A. Börner, E. Schumann, A. Fürste [et al.] // Theor. Appl. 
Genet. - 2002. - Vol. 105, Iss. 6-7. - P. 921-936. doi: 10.1007/s00122-002-0994-1

7. Chromosomal locations and genetic relationships of tiller and spike characters in wheat / W. L. Li, J. C. Nelson, C. Y. Chu [et al.] // Euphytica. - 2002. - Vol. 125, Iss. 3. - P. 357-366. doi: 10.1023/A:1016069809977

8. Identification and mapping of genetic loci affecting the freethreshing habit and spike compactness in wheat (Triticum aestivum L.) / C. Jantasuriyarat, M. I. Vales, C. J. W. Watson, 0. Riera-Lizarazu // Theor. Appl. Genet. - 2004. - Vol. 108, Iss. 2. - P. 261-273. doi: 10.1007/s00122-003-1432-8

9. Markers associated with a QTL for grain yield in wheat under drought / F. M. Kirigwi, M. V. Ginkel, G. Brown-Guedira [et al.] // Mol. Breeding. - 2007. - Vol. 20, Iss. 4. - P. 401-413. doi: 10.1007/s11032-007-9100-3

10. QTL mapping for yield and yield contributing traits in two mapping populations of bread wheat / N. Kumar, P. L. Kulwal, H. S. Balyan, P. K. Gupta // Mol. Breeding. - 2007. - Vol. 19, Iss. 2. - P. 163-177. doi: 10.1007/s11032-006-9056-8

11. Mapping QTL for grain yield, yield components, and spike features in a doubled haploid population of bread wheat / B. Heidari, B. E. Sayed-Tabatabaei, G. Saeidi, K. Suenaga // Genome. - 2011. - Vol. 54, Iss. 6. - P. 517-527. doi: $10.1139 / \mathrm{g} 11-017$

12. Genetic architecture of main effect QTL for heading date in European winter wheat (Triticum aestivum L.) [Електронний ресурс] / C. Zanke, J. Ling, J. Plieske [et al.] // PLoS ONE. - 2014. - Vol. 9, Iss. 11. е113287. - Режим доступу : http://journals. plos.org/plosone/article?id=10.1371/journal. pone.0113287. doi: 10.1371/journal.pone.0113287

13. Методика проведення експертизи сортів пшениці м'якої (Triticum aestivum L. emend. Fiori et Paol.) на відмінність, однорідність і стабільність [Електронний ресурс]. - Режим доступу : http://sops.gov.ua/uploads/files/documents/Metodiki/12.pdf.

14. Литвиненко М. А. Теоретичні основи та методи селекції озимої м'якої пшениці на підвищення адаптивного потенціалу для умов Степу України : автореф. дис. ... д-ра с.-г. наук : спец. 06.01.05. «Селекція рослин» / М. А. Литвиненко ; Ін-т землеробства УААН. - К., 2001. - 46 с.

15. Диференційна здатність методів ідентифікації сортів пшениці за допомогою мікросателітного аналізу та комп'ютерного визначення морфометричних параметрів зерна / 0. 0. Колесник, С. В. Чеботар, О. М. Хохлов, Ю. М. Сиволап // Вісник ОНУ. Серія : Біологія. - Одеса, 2009. - Т. 14, Вип. 14. - С. 27-42.

16. Диференціація сучасних сортів озимої м'якої пшениці півдня України за алельним складом мікросателітних локусів / 0. О. Колесник, С. В. Чеботар, О. М. Хохлов, Ю. М. Сиволап // Зб. наук. праць СГІ - НЦНС. - Одеса : СГІ - НЦНС, 2012. Вип. 19. - С. 47-59.

17. Ідентифікація і реєстрація генотипів м'якої пшениці (Triticum aestivum L.), ячменю (Hordeum vulgare L.), кукурудзи (Zea mays L.), соняшника (Helianthus annuus L.) за допомогою аналізу мікросателітних локусів : метод. рекоменд. / Ю. М. Сиволап, В. В. Волкодав, М. С. Бальвинська [та ін.]. - Одеса : ТОВ «Зовнішрекламсервіс», 2004. - 14 с.

18. Использование ПЦР-анализа в генетико-селекционных исследованиях : науч.-метод. руководство / под ред. Ю. М. Сиволапа. - К. : Аграрна наука, 1998. - С. 34-40.

19. A microsatellite map of wheat / M. S. Röder, V. Korzun, K. Wendehake [et al.] // Genetics. - 1998. - Vol. 149, No. 4. P. 2007-2023. doi: 10.1016/B0-12-227620-5/00113-0

20. Рокицкий П. Ф. Биологическая статистика / П. Ф. Рокицкий. - 3-е изд., испр. - М. : Колос, 1973. - 320 с.

21. Stroup W. W. Removing spatial variation from wheat yield trials: a comparison of methods / W. W. Stroup, P. S. Baenziger, D. K. Mulitze // Crop Science. - 1994. - Vol. 34, No. 1. - P. 62 66. doi: 10.2135/cropsci1994.0011183X003400010011x

22. Landjeva S. Evaluation of genetic diversity among Bulgarian winter wheat (Triticum aestivum L.) varieties during the pe- riod 1925-2003 using microsatellites / S. Landjeva, V. Korzun, G. Ganeva // Gen. Res. Crop Evol. - 2006. - Vol. 53, Iss. 8. P. 1605-1614. doi: 10.1007/s10722-005-8718-4

23. Linkage mapping of four gene loci, Glu-B1, Gli-B1, Rg1 and Yr10 on chromosome 1B of bread wheat / P. I. Payne, L. M. Holt, R. Johnson, J. W. Snape // Genetica Agraria. - 1986. - Vol. 40. - P. 231-242.

24. Khlestkina E. K. Genes determining the coloration of different organs in wheat / E. K. Khlestkina // Russ. J of Genetics: Appl Research. - 2013. - Vol. 3, Iss. 1. - P. 54-65. doi: 10.1134/S2079059713010085

25. Mapping of the Vrn-B1 gene in Triticum aestivum L. using microsatellite markers / I. Leonova, E. Pestsova, E. Salina [et al.] // Plant Breeding. - 2003. - Vol. 122, No. 3. - P. 209-212. doi: 10.1046/j.1439-0523.2003.00818.x

26. Chaturvedi B. K. Selection parameters for some grain and quality attributes in spring wheat (Triticum aestivum L.) / B. K. Chaturvedi, R. R. Gupta // Agricultural Science Digest Kernal. 1995. - Vol. 15, No. 4. - P. 186-190.

\section{References}

1. Röder, M. S., Huang, X.-Q., \& Börner, A. (2008). Fine mapping of the region on wheat chromosome $7 \mathrm{D}$ controlling grain weight. Funct Integr Genomics, 8(1), 79-86. doi: 10.1007/s10142-007-0053-8

2. Gupta, P. K., Rustgi, S., \& Kumar, N. (2006). Genetic and molecular basis of grain size and grain number and its relevance to grain productivity in higher plants. Genome, 49(6), 565-571. doi: 10.1139/g06-063

3. Dilbirligi, M., Eraymana, M., Campbell, B. T., Randhawa, H. S., Baenziger, P. S., Dweikat, I., \& Gill, K. S. (2006). High-density mapping and comparative analysis of agronomically important traits on wheat chromosome 3A. Genomics, 88(1), 74-87. doi: 10.1016/j.ygeno.2006.02.001

4. Litvinenko N. A. (1998). Breeding intensive winter bread wheat varieties for Southern Ukraine. Euphytica, 100(1-3), 7-14. doi: 10.1023/A:1018383317939

5. Brbaklic, L., Trkulja, D., Kondic-Spika, A., Treskic, S., \& Kobiljski, B. (2013). Detection of QTLs for important agronomical traits in hexaploid wheat using association analysis. Czech J. Genet. Plant Breed, 49(1), 1-8.

6. Börner, A., Schumann, E., Fürste, A., Cöster, H., Leithold, B. Röder, M., \& Weber, W. (2002). Mapping of quantitative trait loci determining agronomic important characters in hexaploid wheat (Triticum aestivum L.). Theor. Appl. Genet, 105(6-7), 921936. doi: 10.1007/s00122-002-0994-1

7. Li, W. L., Nelson, J. C., Chu, C. Y., Shi, L. H., Huang, S. H., \& Liu, D. J. (2002). Chromosomal locations and genetic relationships of tiller and spike characters in wheat. Euphytica, 125(3), 357-366. doi: 10.1023/A:1016069809977

8. Jantasuriyarat, C., Vales, M. I., Watson, C. J. W., \& Riera-Lizarazu, 0. (2004). Identification and mapping of genetic loci affecting the free-threshing habit and spike compactness in wheat (Triticum aestivum L.). Theor. Appl. Genet, 108(2), 261273. doi: $10.1007 / \mathrm{s} 00122-003-1432-8$

9. Kirigwi, F. M., Ginkel, M. V., Brown-Guedira, G., Gill, B. S., Paulsen, G. M., \& Fritz, A. K. (2007). Markers associated with a QTL for grain yield in wheat under drought. Mol. Breeding, 20(4), 401413. doi: 10.1007/s11032-007-9100-3

10. Kumar, N., Kulwal, P. L., Balyan, H. S., \& Gupta, P. K. (2007). QTL mapping for yield and yield contributing traits in two mapping populations of bread wheat. Mol. Breeding, 19(2), 163-177. doi: 10.1007/s11032-006-9056-8

11. Heidari, B., Sayed-Tabatabaei, B. E., Saeidi, G., \& Suenaga, K. (2011). Mapping QTL for grain yield, yield components, and spike features in a doubled haploid population of bread wheat. Genome, 54(6), 517-527. doi: 10.1139/g11-017

12. Zanke, C., Ling, J., Plieske, J. Kollers, S., Ebmeyer, E., Korzun, V., \& Röder, M. S. (2014). Genetic architecture of main effect QTL for heading date in European winter wheat (Triticum aestivum L.). PLOS ONE, 9(11), e113287. Retrieved from http://journals. 
plos.org/plosone/article?id=10.1371/journal. pone. 0113287 . doi: 10.1371/journal.pone.0113287

13. Metodyka provedennia ekspertyzy sortiv pshenytsi miakoi (Triticum aestivum L. emend. Fiori et Paol.) na vidminnist, odnoridnist i stabilnist [Regulations on the procedure and the conduct of tests for distinctness, uniformity and stability (DUS) of new bread wheat (Triticum aestivum L. emend. Fiori et Paol.) varieties for the purpose of granting the Breeders' Right]. (n.d.). Retrieved from http://sops.gov.ua/uploads/files/documents/ Metodiki/12.pdf. [in Ukrainian]

14. Lytvynenko, M. A. (2001). Teoretychni osnovy ta metody selektsii ozymoi miakoi pshenytsi na pidvyshchennia adaptyvnoho potentsialu dlia umov Stepu Ukrainy [Theoretical basis and methods of breeding bread winter wheat of universal type for increasing its adaptive ability under the conditions of the Steppe zone of Ukraine]. (Extended Abstract of Dr. Agric. Sci. Diss.). Institute of Agriculture, Kyiv, Ukraine. [in Ukrainian]

15. Kolesnik, 0. 0., Chebotar, S. V., Khokhlov, O. M., \& Sivolap, Yu. M. (2009). Differentiating ability of wheat variety identification methods using microsatellite analysis and computerbased determination of the grain morphometric parameters. Visnyk Odeskoho natsionalnoho universytetu. Biolohiia [0desa National University Herald. Biology], 14(14), 27-42. [in Ukrainian]

16. Kolesnyk, 0. 0., Chebotar, S. V., Khokhlov, 0. M., \& Sivolap, Yu. M. (2012). Differentiation of the modern soft winter wheat varieties from the south of Ukraine by allele composition of microsatellite loci. Zbirnyk naukovykh prats SHI-NTSNS [Collected scientific articles of PBGI-NCSCI], 19(59), 47-59. [in Ukrainian]

17. Syvolap, Yu. M., Volkodav, V. V., \& Balvynska, M. S. (2004). Identyfikatsiia i reiestratsiia henotypiv miakoi pshenytsi (Triticum aestivum L.), yachmeniu (Hordeum vulgare L.), kukurudzy (Zea mays L.), soniashnyka (Helianthus annuus L.) za dopomohoiu analizu mikrosatelitnykh lokusiv [Identification and registration of genotypes of common wheat (Triticum aestivum L.), barley (Hordeum vulgare L.), maize (Zea mays L.) and sunflower (Helianthus annuus L.) by microsatellite locus analysis]. Odesa: TOV "Zovnishreklamservis". [in Ukrainian]

18. Syvolap, Yu. M. (Ed.). (1998). Ispol'zovanie PTsR-analiza $v$ genetiko-selektsionnykh issledovaniyakh [The Use of PCR-analysis in Genetic and Breeding Research]. Kyiv: Ahrarna nauka. [in Russian]

19. Röder, M. S., Korzun, V., Wendehake, K., Plaschke, J., Tixier, M. H., Leroy, P., \& Ganal, M. W. (1998). A microsatellite map of wheat. Genetics, 149(4), 2007-2023. doi: 10.1016/B0-12-227620-5/00113-0

20. Rokitskiy, P. F. (1973). Biologicheskaya statistika [Biological Statistics]. Moscow: Kolos. [in Russian]

21. Stroup, W. W., Baenziger, P. S., \& Mulitze, D. K. (1994). Removing spatial variation from wheat yield trials: a comparison of methods. Crop Science, 34(1), 62-66. doi: 10.2135/cropsci1994.0011183X003400010011x

22. Landjeva, S., Korzun, V., \& Ganeva, G. (2006). Evaluation of genetic diversity among Bulgarian winter wheat (Triticum aestivum L.) varieties during the period 1925-2003 using microsatellites. Gen. Res. Crop Evol, 53(8), 1605-1614. doi: 10.1007/s10722-005-8718-4

23. Payne, P. I., Holt, L. M., Johnson, R., \& J. W. Snape, (1986). Linkage mapping of four gene loci, Glu-B1, Gli-B1, Rg1 and Yr10 on chromosome 1B of bread wheat. Genetica Agraria, 40, 231-242.

24. Khlestkina, E. K. (2013). Genes determining the coloration of different organs in wheat. Russ. J. of Genetics: Appl Research, 3(1), 54-65. doi: 10.1134/S2079059713010085

25. Leonova, I., Pestsova, E., Salina, E. Efremova T., Röder, M. S., Börner, A., \& Fischbeck, G. (2003). Mapping of the Vrn-B1 gene in Triticum aestivum L. using microsatellite markers. Plant Breeding, 122(3), 209-212. doi: 10.1046/j.1439-0523.2003.00818.x

26. Chaturvedi, B. K., \& Gupta, R. R. (1995). Selection parameters for some grain and quality attributes in spring wheat (Triticum aestivum L.). Agric. Sci. Digest. Kernal, 15(4), 186-190.

\section{УДК 577.2:631[633.111:664.64.016.8]}

Колесник 0. 0. ${ }^{*}$, Хохлов 0. М., Чеботар С. В. Асоціації алелів мікросателітних маркерів з агрономічними ознаками сучасних сортів пшениці м'якої озимої Півдня України // Сортовивчення та охорона прав на сорти рослин. - 2016. - № 3. - С. 19-29. http://dx.doi.org/10.21498/2518-1017.3(32).2016.75973

Селекційно-генетичний інститут - Національний центр насіннєзнавства та сортовивчення, вул. Овідіопольська дорога, 3, м. 0деса, 65036, Украина, "e-mail: emerald-olga@ukr.net

Мета. Визначення асоціацій мікросателітних маркерів зі специфічними областями геному, які контролюють важливі агрономічні ознаки, на виборці сортів пшениці м'якої озимої селекції СГІ - НЦНС, зареєстрованих в Державному реєстрі сортів, придатних для поширення в Україні, протягом різних років. Методи. Молекулярногенетичні методи (виділення геномної ДНК, полімеразна ланцюгова реакція (ПЛР), електрофорез продуктів ампліфікації в поліакриламідному гелі), польові методи (фенологічні спостереження дати колосіння та аналіз ознак «висота рослин», «довжина колосу» та «довжина остюків», візуальні - оцінка ознаки «колір колосу»), статистичні методи (визначення середніх значень агрономічних ознак за допомогою описової статистики з використанням програми Excel iз пакету Microsoft Office, дисперсійний аналіз виконано процедурою GLM (гене- ральна лінійна модель) зі спеціалізованого програмного комплексу AGROBASE 21). Результати. Протягом чотирьох років (2010/11, 2011/12, 2012/13, 2013/14) 47 сортів пшениці м'якої озимої, були вивчені за фенотиповими ознаками та проаналізовані за 17 мікросателітними локусами. Виявлено 35 асоціацій маркерів з датою колосіння, 39 - з висотою рослин, 33 - з розміром остей, $20-3$ кольором колосу і 8 - 3 розміром колосу; асоціації були стабільними та достовірними протягом 2-4 років дослідження. Висновки. Мікросателітні маркери, які виявили значні та стабільні в різні роки асоціації 3 агрономічними ознаками, можуть бути корисними й придатними для добору за допомогою маркерів (MAS) у рамках програм селекції пшениці України.

Ключові слова: асоціації маркер-ознака, Triticum aestivum L., сорти пшениці м'якої озимої. 
УДК 577.2:631[633.111:664.64.016.8]

Колесник О. А. ${ }^{*}$, Хохлов А. Н., Чеботарь С. В. Ассоциации аллелей микросателлитных маркеров с агрономическими признаками современных сортов пшеницы мягкой озимой Юга Украины // Сортовивчення та охорона прав на сорти рослин. - 2016. - № 3. - C. 19-29. http://dx.doi.org/10.21498/2518-1017.3(32).2016.75973

Селекционно-генетический институт - Национальный центр семеноведения и сортоизучения, ул. Овидиопольская дорога, 3 , 2. 0десса, 65036, Украина, ${ }^{*}$ e-mail: emerald-olga@ukr.net

цель. Определение ассоциаций микросателлитных маркеров со специфическими областями генома, которые контролируют важные агрономические признаки, на выборке сортов пшеницы мягкой озимой селекции СГИ - НЦНС, зарегистрированных в Государственном реестре сортов растений, пригодных для выращивания в Украине, на протяжении разных лет. Методы. Молекулярно-генетические методы (выделение геномной ДНК, полимеразная цепная реакция (ПЦР), электрофорез продуктов амплификации в полиакриламидном геле), полевые методы (фенологические наблюдения даты колошения и анализ признаков «высота растений», «длина колоса» и «длина остей», визуальные - оценка признака «цвет колоса»), статистические методы (определение средних значений агрономических признаков с помощью описательной статистики с использованием программы Excel из пакета Microsoft Office, дисперсионный анализ выполнен процедурой GLM (генеральная линейная модель) из специализированного программного комплекса AGROBASE 21). Результаты. В течение четырех лет $(2010 / 11,2011 / 12,2012 / 13,2013 / 14) 47$ сортов пшеницы мягкой озимой были изучены по фенотипическим признакам и проанализированы с помощью 17 микросателлитных локусов. Выявлено 35 ассоциаций маркеров с датой колошения, 39 - с высотой растений, 33 - с размером остей, 20 - с цветом колоса и 8 - с размером колоса; ассоциации были стабильными и достоверными на протяжении 2-4 лет исследования. Выводы. Микросателитные маркеры, которые обнаружили значительные и стабильные в разные годы ассоциации с агрономическими признаками, могут быть полезными и пригодными для отбора с помощью маркеров (MAS) в рамках программ селекции пшеницы Украины.

Ключевые слова: ассоциации маркер-признак, Triticum aestivum L., copта пшеницы мягкой озимой.

Надійшла 16.02.2016 